\title{
Puntuación diagnóstica para apendicitis: estudio prospectivo de su aplicación por profesionales de salud no-médicos
}

\author{
Marcelo A Beltrán¹,2, Raúl Villar M², Karina S Cruces ${ }^{1 a}$. \\ Application of a diagnostic score for \\ appendicitis by health-related non- \\ physician professionals
}

Background: Diagnostic scores have been developed as simple, user-friendly, cost-effective instruments to improve the early diagnosis of acute appendicitis. Aim: To assess the yield of a diagnostic score for acute appendicitis, measured by trained health-related non-physician professional. Patients and methods: A prospective, double blind, non-randomized trial. Emergency room nurses applied a previously validated diagnostic score for appendicitis to patients aged more than 15 years with abdominal pain and tachycardia or fever. The main outcome was the percentage of patients operated for acute appendicitis that had a positive score for appendicitis and a pathologically demonstrated appendicitis. Results: The mean scores for patients operated or not operated for appendicitis were 8.64 and 3.31, respectively. The figures for sensitivity, specificity and diagnostic accuracy of the score were $0.83,0.98$ and 0.94 , respectively. Conclusions: An appendicitis score measured by healthrelated non-physician professionals has a similar diagnostic yield than clinical judgment (Rev Méd Chile 2006; 134: 39-47).

(Key words: Appendicitis; Diagnosis; Diagnostic techniques and procedures; Emergency room nursing)

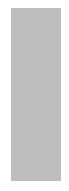

$\mathrm{E}$ diagnóstico precoz y acertado de apendicitis aguda (AA) ha sido una preocupación constante para los cirujanos, desde los albores de la cirugía moderna. Reginald Fitz, en $1886^{1}$, describió el proceso patológico de la apendicitis y

Correspondencia a: Dr. Marcelo A Beltrán. Casilla \#308. Plazuela Baquedano 240. Teléfono: +0562 53637055. Ovalle, IV Región, Chile. E mail: beltran_01@yahoo.com recomendó su diagnóstico y tratamiento precoz. Esta recomendación no ha cambiado en 120 años. El diagnóstico de AA es relativamente simple cuando se presenta el cuadro clínico clásico ${ }^{2-8}$. La anamnesis y el examen físico constituyen las modalidades diagnósticas más prácticas y efectivas $^{2-9}$. Las dificultades se encuentran en el reconocimiento de las presentaciones atípicas de $\mathrm{AA}^{2-7}$. Con el objetivo de mejorar el diagnóstico 
precoz de AA y reducir las tasas de diagnósticos tardíos y falsos, se introdujeron técnicas radiológicas, estudios de laboratorio y laparoscopia ${ }^{9-26}$. A pesar de este abordaje tecnológico todavía se operan apéndices normales y perforados ${ }^{3,15}$. Estas consideraciones han llevado al desarrollo de puntuaciones diagnósticas de AA, diseñadas para mejorar el diagnóstico y tratamiento precoz de AA y reducir las tasas de apendicectomías en blanco y perforadas ${ }^{6,27-36}$, ambas circunstancias tienen consecuencias en términos de complicaciones, costos e implicaciones legales ${ }^{3,37}$. La más conocida de estas puntuaciones es el Score de Alvarado (SA) ${ }^{32}$. Tiene las ventajas de su simplicidad, es fácilmente comprensible, no-invasivo y costo-efectivo ${ }^{31-33,38}$. El gran número de escalas y puntuaciones diagnósticas para $\mathrm{AA}$, desarrolladas en diferentes instituciones ${ }^{27-36}$, demuestra la elevada incertidumbre para el diagnóstico de AA que prevalece entre los médicos de las unidades de emergencias (UE). La utilidad demostrada por estas puntuaciones se debe al hecho de que los médicos deben enfocarse en la información clínica específica disponible e incorporarla en el análisis del paciente ${ }^{4}$.

Las UE son consideradas la fuente primaria de atención médica en muchos países ${ }^{2-6}$. Como consecuencia, los médicos de las UE se ven enfrentados a una gran cantidad de pacientes con dolor abdominal ${ }^{2,3}$. En la UE de nuestra institución, utilizamos una versión modificada del SA, a la cual denominamos puntuación diagnóstica para apendicitis aguda (PDA) y que ha sido previamente validada 6 . Esta escala demostró una elevada sensibilidad y especificidad para el diagnóstico correcto de AA. Consideramos que la PDA es útil en la rápida diferenciación de pacientes con posible AA de aquellos que consultan por otras causas de dolor abdominal, enfoca la evaluación clínica en la posible patología, identifica pacientes que pueden ser derivados directamente al pabelón de operaciones sin estudios de imagen $u$ observación prolongada, e identifica pacientes quienes pueden ser dados de alta en forma segura. El objetivo de este estudio es evaluar la exactitud diagnóstica de la PDA, aplicada por profesionales de salud no-médicos, para el diagnóstico de AA durante el proceso de selección en pacientes que se presentan con dolor abdominal en nuestra UE.

\section{PACIENTES Y MÉTOdOS}

Se diseñó un estudio prospectivo, doble ciego, no aleatorio. Los criterios del German Study Group of Abdominal Pain 29-31,34, fueron el gold standard que utilizamos en el análisis del estudio. En el diseño del estudio seguimos la iniciativa STARD ${ }^{39}$. Los pacientes fueron estudiados en la UE de un hospital con un censo anual de 60.000 consultas de adulto. Aproximadamente 9.000 de esas consultas son por dolor abdominal, de ellas entre 750 y 800 fueron operadas por AA. El estudio se condujo entre mayo de 2003 y noviembre de 2004. Una muestra de pacientes mayores de 15 años de edad fue seleccionada basándose en los siguientes criterios: a) Síntomas abdominales: dolor abdominal asociado a náusea y vómito. b) Dos componentes del síndrome de respuesta inflamatoria sistémica: taquicardia y fiebre. En los pacientes seleccionados se obtuvo un hemograma y se aplicó la PDA. Se excluyeron pacientes menores de 15 años, pacientes con dolor abdominal leve (según escala visual análoga de dolor: leve 0 a 4 puntos, moderado 5 a 7 puntos, severo 8 a 10 puntos), o si existía una causa evidente de dolor abdominal (hernia complicada, etc.). Se eligieron intencionalmente amplios criterios de inclusión para asegurar la generalidad de la información.

Recolección y procesamiento de la información. La información fue recolectada uno de cada 6 días durante $24 \mathrm{~h}$. Dos de los investigadores (MAB y RVM) se encontraron presentes durante las $24 \mathrm{~h}$; las enfermeras de la UE se encontraban presentes en dos períodos de $12 \mathrm{~h}$ durante las $24 \mathrm{~h}$. Por lo tanto, el $100 \%$ de los pacientes elegibles durante las $24 \mathrm{~h}$ del período de estudio fueron incluidos. Los investigadores y las enfermeras, evaluaron individualmente a los pacientes en un área de observación. Cada paciente fue incluido en dos cuestionarios estructurados similares, que fueron llenados uno por los investigadores y otro por las enfermeras. La información recolectada incluyó características demográficas, variables clínicas, diagnóstico y valores de la PDA. El proceso de evaluación consistió en dos fases después de la selección inicial. En la primera fase, se asignó el diagnóstico de abdomen agudo a los pacientes seleccionados sin especificar el posible origen. En el área de observación se tomó un hemograma, con los resultados las enfer- 
meras aplicaron la PDA; las enfermeras fueron previamente entrenadas hasta adquirir la destreza clínica necesaria para medir correctamente la PDA. Durante la segunda fase, el cirujano evaluó a los pacientes 30 min a $3 \mathrm{~h}$ después de que la PDA fuera aplicada. El cirujano formuló un diagnóstico presuntivo y decidió la necesidad de cirugía basándose en su juicio clínico sin tener conocimiento del resultado de la PDA. El cirujano no conoció el resultado de la PDA o el informe histopatológico del apéndice, sino hasta finalizado el estudio.

Puntuación diagnóstica de apendicitis. El SA original $^{31}$ tiene 8 componentes, dos con valor de 2 puntos y los demás con valor de 1 punto, para una puntuación máxima posible de 10 puntos. Realizamos tres modificaciones: Primera, disminuimos el valor asignado a la leucocitosis de 2 puntos a 1 punto. Segunda, eliminamos la «desviación a la izquierda de los leucocitos» y la reemplazamos por sausencia de dolor similar previo ${ }^{4}$. Tercera, añadimos el componente de «dolor al movimiento $\%$. La PDA aún tiene un total posible de 10 puntos. Basándonos en estudios previos $^{6}$, la PDA se dividió en 3 niveles, de 0 a 4 puntos: negativo para AA; de 5 a 7 puntos el paciente debe ser observado por posible AA; y de 8 a 10 puntos se asigna el diagnóstico de AA (Tabla 1).
Como seguimiento a los pacientes evaluados con 5 a 7 puntos que fueron dados de alta, se realizó una visita al día siguiente. Estos pacientes fueron evaluados por otros médicos de la UE que no participaron del estudio, ellos no midieron la PDA o tuvieron conocimiento del resultado previo. Las enfermeras de la UE recolectaron la información y resultados de esta segunda evaluación.

Definiciones. Se aplicaron definiciones establecidas para la historia clínica clásica de $\mathrm{AA}^{2-9}$, variables de la PDA y examen físico. La historia clásica de AA se definió como un episodio que se inicia con dolor epigástrico, periumbilical o difuso, que cambia a dolor localizado en la fosa ilíaca derecha (FID), se acompaña de fiebre de bajo grado, anorexia, náusea y vómito. Habitualmente el paciente no ha expenimentado síntomas similares previamente. La anorexia es la pérdida de apetito después del inicio del dolor. La náusea y vómito se presentan después del inicio del dolor. La fiebre es la elevación subjetiva y objetiva de la temperatura basal del paciente. El dolor al movimiento es aquel que se exacerba con movimientos como: tos, caminar, elevación o flexión de la piema e incluye los signos del psoas y obturador. El dolor de rebote, es el que percibe el paciente cuando durante el examen del abdomen, la

\section{Tabla 1. Variables de la puntuación} Cohorte (n: 850 - 100\%)

\begin{tabular}{|c|c|c|c|c|c|}
\hline \multirow[b]{2}{*}{ Variables de la puntuación } & \multirow[b]{2}{*}{ Puntos } & \multicolumn{2}{|c|}{$\begin{array}{c}\text { Apendicitis } \\
\text { (n: } 207-24,3 \%)\end{array}$} & \multicolumn{2}{|c|}{$\begin{array}{l}\text { No apendicitis } \\
\text { (n: } 643-75,7 \% \text { ) }\end{array}$} \\
\hline & & $\mathrm{n}$ & $\%$ & $\mathrm{n}$ & $\%$ \\
\hline Dolor sobre el punto de Mc Burney & 2 & 198 & 95,7 & 76 & 11,8 \\
\hline Dolor de rebote & 1 & 188 & 90,8 & 177 & 27,5 \\
\hline Migración del dolor & 1 & 169 & 81,6 & 0 & 0 \\
\hline Dolor al movimiento & 1 & 203 & 98,1 & 278 & 43,2 \\
\hline Ausencia de dolor similar previo & 1 & 202 & 97,6 & 282 & 43,9 \\
\hline Anorexia & 1 & 162 & 78,3 & 401 & 62,4 \\
\hline Náusea/vómito & 1 & 166 & 80,2 & 483 & 75,1 \\
\hline Temperatura rectal $>37,5^{\circ} \mathrm{C}$ & 1 & 137 & 66,2 & 139 & 21,6 \\
\hline Leucocitosis & 1 & 173 & 83,6 & 172 & 26,7 \\
\hline Total/Promedio \pm DS & 10 & $177,5 \pm 7,3$ & $85,8 \pm 3,6$ & $223 \pm 51,2$ & $37,4 \pm 8$ \\
\hline Exclusión (negativo para apendicitis) & $0-4$ & 0 & 0 & 450 & 70 \\
\hline Observación (posible apendicitis) & $5-7$ & 48 & 23,2 & 185 & 28,7 \\
\hline Cirugía (apendicitis aguda) & $8-10$ & 159 & 76,8 & 8 & 1,3 \\
\hline
\end{tabular}

DS: Desviación estándar. 
mano del examinador deprime la pared abdominal más de $1 \mathrm{~cm}$ del nivel habitual, durante 30 a $60 \mathrm{~s}$, y súbitamente retira la mano. El signo de Mc Bumey, es el dolor localizado sobre el punto de Mc Bumey que se produce cuando el examinador deprime este punto específico. Se define como leucocitosis al recuento de leucocitos superior a 11.000 por $\mathrm{mm}^{3}$. Se incluyen en el grupo de AA sólo a los pacientes con histopatología positiva para AA.

Análisis estadístico. El resultado principal fue el porcentaje de pacientes operados por AA que tuvieron una PDA positiva para AA y AA histopatológicamente demostrada. El resultado secundario fue el porcentaje de pacientes operados por AA con PDA positiva para AA, que no tenían AA histopatológicamente demostrada. El universo de estudio fue seleccionado sobre la base de una población conocida de 1.200 pacientes. Para un valor aaa 0,01 , error $10 \%$, poder $80 \%$, intervalos de confianza (IC) $95 \%$ y nivel estadístico de $\mathrm{p}<0,05$, se requirió una muestra de 166 pacientes. Las variables continuas fueron analizadas con el test tStudent. Las variables categóricas se presentan como promedio y desviación standard (DS); se empleó el test $\mathrm{Chi}^{2}$ para su análisis. Se midió la sensibilidad, especificidad, valores predictivos negativo (VPN) y positivo (VPP) y exactitud diagnóstica (ED) del score. El análisis estadístico fue realizado con el programa SPSS (Statistical Package for Social Sciences, 11.0; Chicago, Illinois).

\section{RESULTADOS}

Se estudiaron 850 pacientes. De ellos, 207 (24,3\%) fueron operados por AA. La mayoría de los componentes de la PDA se encontraban presentes en la mayoría de los pacientes operados por AA. En el grupo de pacientes sin AA, algunos componentes de la PDA no se encontraban presentes o los presentaba una pequeña proporción de pacientes (Tabla 1). De los 207 pacientes operados por AA, 83 (40\%) eran hombres y 124 (60\%) mujeres; la edad promedio fue 30,24 años (Tabla 2). Los síntomas que presentaron los pacientes con AA fuemon: dolor abdominal inicialmente epigástrico, periumbilical 0 difuso: 173 (83,6\%), migración del dolor a FID: 169 (81,6\%), náusea y vómito: 166 (80,2\%), anorexia: 162 $(78,3 \%)$ y fiebre: 159 (76,8\%). Los hallazgos del examen físico fueron: dolor al movimiento: 203 (98,1\%), dolor al toser. 200 (96,6\%), signo de Mc

Tabla 2. Demografía

\begin{tabular}{|c|c|c|c|c|c|}
\hline \multirow{2}{*}{\multicolumn{2}{|c|}{ Sexo }} & \multicolumn{2}{|c|}{$\begin{array}{c}\text { Apendicitis (n: 207-24,3\%) } \\
\text { Hombre/Mujer (\%): 83/124 (40/60) }\end{array}$} & \multicolumn{2}{|c|}{$\begin{array}{c}\text { No apendicitis (n: } 643-75,7 \%) \\
\text { Hombre/Mujer (\%): } 169 / 474(26,3 / 73,7)\end{array}$} \\
\hline & & $\mathrm{n}$ & $\%$ & $\mathrm{n}$ & $\%$ \\
\hline \multirow[t]{3}{*}{ Edad } & $15-30$ & 116 & 56 & 181 & 28,1 \\
\hline & $31-60$ & 86 & 41,5 & 333 & 51,7 \\
\hline & $>61$ & & 2,5 & 129 & 20,2 \\
\hline \multicolumn{2}{|c|}{ Promedio \pm DS } & \multicolumn{2}{|c|}{$30,24+0,862(15-79)$} & \multicolumn{2}{|c|}{$43,31 \pm 0,728$ (15-91) } \\
\hline
\end{tabular}

DS: Desviación estándar.

Tabla 3. Valores de la puntuación

\begin{tabular}{|lcccccc|}
\hline \multirow{2}{*}{ Nivel } & \multicolumn{2}{c}{$\begin{array}{c}\text { Apendicitis (n:207) } \\
\text { n }\end{array}$} & \multicolumn{2}{c}{ No apendicitis (n:643) } & & IC $95 \%$ \\
\hline $0-4$ & 0 & 0 & 450 & 70 & 0,001 & $0 / 256,4$ \\
$5-7$ & 48 & 23,2 & 185 & 28,7 & 0,044 & $0,4 / 0,9$ \\
$8-10$ & 159 & 76,8 & 8 & 1,3 & 0,027 & $4,7 / 1,8$ \\
Total & 207 & 100 & 643 & 100 & 0,490 & $-892 / 601$ \\
Promedio \pm DS & $8,64 \pm 0,094(5-10)$ & $3,31 \pm 0,085(0-9)$ & 0,032 & $7,5 / 2,6$ \\
\hline
\end{tabular}

IC: Intervalos de confianza, DS: Desviación estándar. 
Burney: 198 (95,7\%), dolor de rebote: 188 (91\%), defensa muscular: $181(87,4 \%)$ y signo de Rovsing: 49 (23,7\%). Ningún paciente operado por AA tuvo un puntaje de 40 menor. La mayoría de los pacientes operados por AA tenían un puntaje de 80 mayor. El puntaje promedio para pacientes con AA fue 8,64, para pacientes sin AA, el puntaje promedio fue 3,31 (Tabla 3). El puntaje promedio para AA es comparable al puntaje promedio de pacientes con úlcera duodenal perforada (Tabla 4). La distribución y frecuencia de la PDA en ambos grupos de pacientes se compara en la Figura 1. La mayor parte

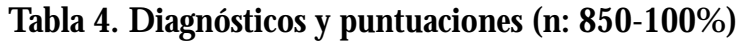

\begin{tabular}{|lcccc|}
\hline Diagnóstico & $\mathrm{n}$ & $\%$ & Puntuación promedio & $\mathrm{DS} \pm$ \\
\hline Apendicitis & 207 & 24,3 & $8,64(5-10)$ & 0,094 \\
Cólico biliar & 130 & 15,3 & $2,55(0-5)$ & 0,135 \\
Gastroenteritis aguda & 108 & 12,7 & $4,09(1-7)$ & 0,154 \\
Síndrome de intestino irritable & 84 & 10 & $0,20(0-2)$ & 0,066 \\
Gastritis & 64 & 7,5 & $2,30(1-5)$ & 0,124 \\
Colecistitis aguda & 58 & 7 & $3,79(1-6)$ & 0,147 \\
Cólico renal & 53 & 6,3 & $2,92(2-4)$ & 0,054 \\
Infecciones urinarias & 47 & 5,5 & $6,23(1-7)$ & 0,190 \\
Patología ginecológica & 23 & 3 & $4,68(2-6)$ & 0,297 \\
Colangitis & 22 & 2,7 & $5,73(4-6)$ & 0,135 \\
Pancreatitis aguda & 18 & 2 & $4,65(3-6)$ & 0,209 \\
Ulcera duodenal perforada & 11 & 1,3 & $8,14(6-9)$ & 0,553 \\
Obstrucción intestinal & 8 & 1 & $7(7)$ & 0,000 \\
Diverticulitis & 5 & 0,7 & $4,01(1-5)$ & 1,000 \\
Tumor abdominal & 3 & 0,3 & $2,67(2-4)$ & 0,667 \\
Trauma abdominal antiguo & 3 & 0,3 & $2,67(2-4)$ & 0,667 \\
Neumonía & 3 & 0,3 & $5(5)$ & 0,000 \\
Misceláneas* & 3 & 0,3 & $3,33(1-6)$ & 1,453 \\
Total & 850 & 100 & $4,61(0-10)$ & 0,104 \\
\hline
\end{tabular}

* Isquemia mesentérica, pancreatitis crónica y herpes zoster: 1 paciente

DS: Desviación estándar.

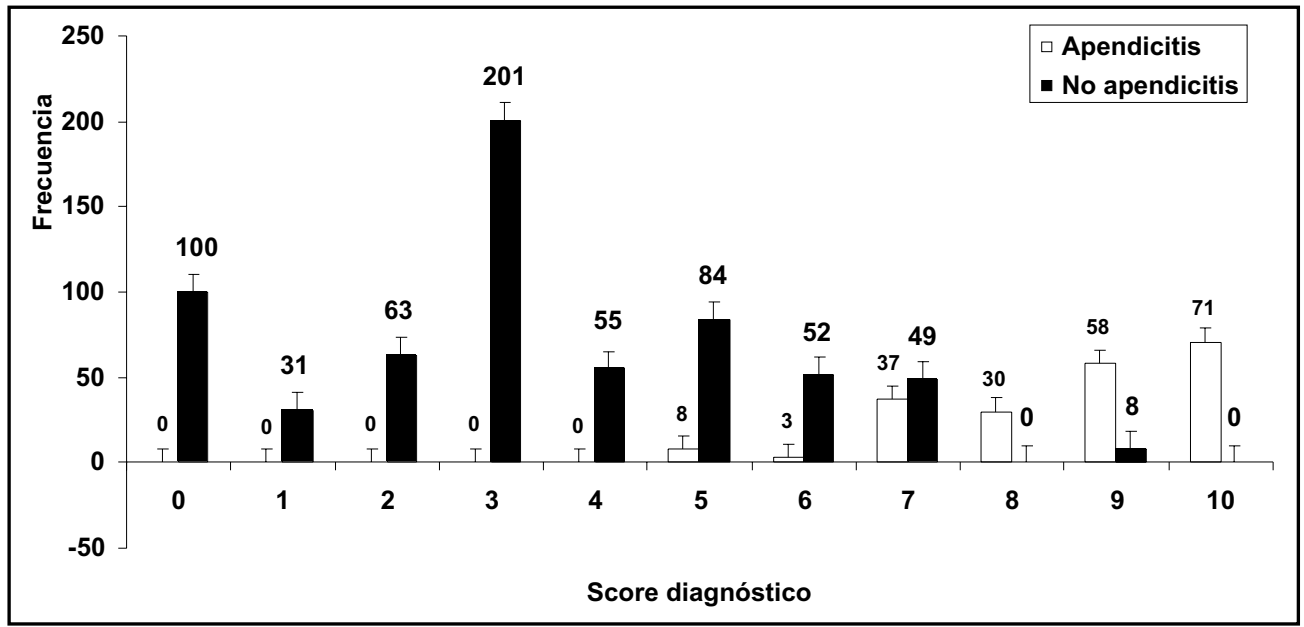

FiguRA 1. Distribución de la frecuencia según el score en pacientes con y sin apendicitis.

Comparación de la frecuencia de distribución de acuerdo al score diagnóstico en pacientes con y sin apendicitis. Un score de 7 se encontraba presente en una proporción similar de pacientes en ambos grupos. 
de los pacientes sin AA tuvo una PDA de 6 o menor, una proporción similar de pacientes en ambos grupos tuvo una PDA de 7.

El diagnóstico quirúrgico se describe en la Tabla 5, en pacientes con peritonitis difusa 0 flegmón, el promedio de la PDA fue menor que en pacientes con apendicitis, peritonitis localizada 0 absceso. Según la histopatología: 82,3\% de los apéndices no se encontraban perforados, $4,2 \%$ fueron normales y $13,5 \%$ perforados. La AA se diagnostica dentro de las primeras $48 \mathrm{~h}$ de evolución; 160 (77,3\%) pacientes fueron diagnosticados dentro de ese período (promedio 38,86 \pm 2,246, IC 95\% -34,66/-25,79 p <0,001, Figura 2).
La PDA tuvo una sensibilidad de 0,83 , especificidad 0,98 y ED 0,94 (Tabla 6). Se operaron 159 pacientes por AA con PDA de 8 a 10 puntos, todos ellos tenían AA histopatológicamente demostrada. Ningún paciente se operó por AA con PDA mayor a 8 puntos que tuviera otra patología 0 apéndice normal en la histopatología. La tasa de apendicitis perforada fue $13,5 \%$. Nueve pacientes con PDA de 5 a 7 puntos tenían el apéndice normal, de ellos 2 tenían procesos patológicos de origen ginecológico. Un total de 185 pacientes con PDA de 5 a 7 no fueron operados, todos ellos fueron reevaluados al día siguiente de la medición de la PDA durante una

Tabla 5. Diagnóstico quirúrgico en pacientes con apendicitis (n: 207)

\begin{tabular}{|lrrc|}
\hline Diagnóstico & $\mathrm{n}$ & $\%$ & Score $\pm \mathrm{DS}$ \\
\hline Apendicitis & 168 & 81,2 & $8,7 \pm 1,316(5-10)$ \\
Peritonitis localizada & 17 & 8,2 & $9 \pm 0,707(8-10)$ \\
Peritonitis difusa & 11 & 5,3 & $7,91 \pm 1,044(7-9)$ \\
Flegmón & 8 & 3,9 & $6,75 \pm 1,982(5-9)$ \\
Absceso & 3 & 1,4 & $10 \pm 0,000(10)$ \\
\hline
\end{tabular}

DS: Desviación estándar.

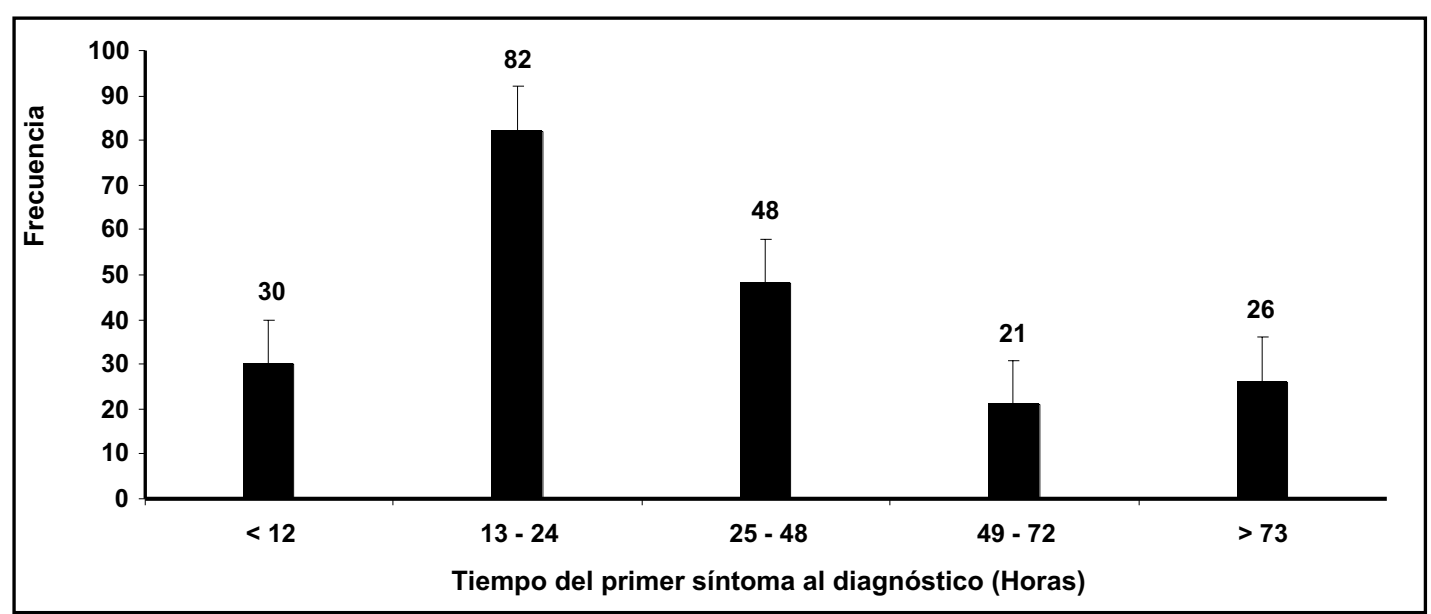

Figura 2. Evolución clínica del cuadro de apendicitis aguda.

La apendicitis aguda se diagnostica habitualmente dentro de las primeras $48 \mathrm{~h}$ del inicio de los síntomas. El período promedio desde los síntomas iniciales hasta el diagnóstico fue de 38,86 h (7-48) en pacientes con apendicitis. 
Tabla 6. Sensibilidad, especificidad y exactitud diagnóstica

\begin{tabular}{|lccccccccc|}
\hline Score & Sensibilidad & Especificidad & LR (+) & LR (-) & VPP & VPN & ED & P & PP \\
\hline $0-4$ & 0,00 & 1,00 & 0,00 & 0,00 & 0,00 & 1,00 & 0,00 & 0,00 & 0,00 \\
$5-7$ & 0,50 & 0,95 & 1,25 & 0,52 & 0,81 & 0,83 & 0,82 & 0,33 & 0,37 \\
$8-10$ & 0,95 & 0,95 & 2,37 & 0,04 & 0,95 & 0,95 & 0,95 & 0,50 & 0,54 \\
$0-10$ & 0,83 & 0,98 & 8,3 & 0,16 & 0,95 & 0,94 & 0,94 & 0,25 & 0,73 \\
IC $95 \%$ & $-110,3 / 30,3$ & $-64 / 250,6$ & $-104,6 / 54$ & & \multicolumn{2}{c}{$-50,2 / 2,2$} \\
\hline
\end{tabular}

IC: Intervalo de confianza, LR: Likelihood rate, VPP: Valor predictivo positivo, VPN: Valor predictivo negativo, ED: Exactitud diagnóstica, P: Prevalencia (Probabilidad pre test), PP: Probabilidad post test.

visita de seguimiento por el cirujano de la UE de turno. Ningún paciente con PDA de 5 a 7 puntos fue operado durante las siguientes $24 \mathrm{~h}$ después que fueran evaluados por nuestro grupo.

\section{DISCUSIÓN}

A pesar del abordaje tecnológico actual para el diagnóstico de una patología tan común como la $\mathrm{AA}^{9-26}$, su diagnóstico temprano y acertado es todavía un problema y un desafín ${ }^{3,7,31}$. Las tasas de perforación, apéndices normales y laparotomías negativas varían entre $15 \%$ a $40 \%$ 4-7,31,34. Algunos pacientes se presentan con síntomas atípicos que pueden confundir a médicos inexpertos y a médicos con amplia experiencia ${ }^{2,3}$. En muchos lugares, la primera consulta por AA se realiza en una posta rural o en un consultorio sin médico residente, estos pacientes son evaluados por enfermeras o auxiliares de enfermería. Los errores de diagnóstico y manejo son frecuentes. Los métodos de diagnóstico más importantes para AA son la anamnesis y examen físico ${ }^{2-9}$. Algunas de las técnicas de diagnóstico por imagen reportadas no se encuentran ampliamente disponibles ${ }^{13,17}$. En muchos hospitales, aun los exámenes más simples no se encuentran disponibles las $24 \mathrm{~h}$ del día 2,6,10,11,14-18, o no se relacionan con la presentación clínica y hallazgos de laboratorio ${ }^{9,15}$. Las puntuaciones diagnósticas de AA han demostrado su valor cuando se incorporan en la práctica clínica rutinaria ${ }^{27-36}$. Este estudio fue diseñado con el propósito de probar la exactitud diagnóstica y la real utilidad de la PDA aplicada por profesionales de la salud no-médicos. Se diseñó el estudio de manera tal que ningún médico midiera o aplicara la PDA. Por lo tanto se midieron las habilidades y experiencia del médico contra la performance de la PDA medida por profesionales de salud no-médicos.

Se encontró una concordancia de 100\% entre el juicio clínico del cirujano y la PDA para identificar pacientes sin AA (0-4 puntos). En 167 pacientes, la PDA fue de 8 a 10 puntos, el cirujano decidió que 159 (95,2\%) de ellos debían ser operados por AA, el informe histopatológico confirmó el diagnóstico. Otros pacientes que tenían una PDA de 8 a 10 puntos, presentaron un cuadro quirúrgico severo: úlcera duodenal perforada en 8 casos. La elevada ED de la PDA demuestra que el uso de este instrumento clínico permite que profesionales de salud no-médicos, entrenados en su aplicación puedan diagnosticar presuntamente una AA con exactitud similar al juicio clínico de un cirujano.

La tasa de apendicectomía negativa fue 4,2\%, la tasa de laparotomía negativa $3,4 \%$ y la tasa de AA perforada $13,5 \%$; comparables a los valores esperados para estudios clínicos de diagnóstico de $\mathrm{AA}^{29-31}$. Las limitaciones de este estudio son: 1) No se hizo seguimiento por más de $24 \mathrm{~h}$ a los pacientes con PDA de 5 a 7 puntos dados de alta de la UE, consiguientemente no fue posible evaluar la evolución de estos pacientes más allá de las $24 \mathrm{~h}$ de su visita inicial. 2) No incluimos en el estudio los 7 días de la semana, consiguientemente capturamos aproximadamente $15 \%$ de las visitas semanales de pacientes adultos a la UE. 


\section{CONCLUSIONES}

La PDA medida por profesionales de la salud no-médicos es similar al criterio médico. La aplicación de la PDA puede encontrar su utilidad en el proceso de selección y diagnóstico

\section{REFERENCIAS}

1. Fitz RH. Perforating information of the vermiform appendix, with special reference to its early diagnosis and treatment. Am J Med Sci 1886; 92:321-46.

2. Beltrán MS, Viliar RM, Tapia TF, Cruces KB. Sintomatología atípica en 140 pacientes con apendicitis. Rev Chil Cir 2004; 56: 269-74.

3. Von Titte SN, McCabe CJ, Ottinger LW. Delayed appendectomy for appendicitis: Causes and consequences. Am J Emerg Med 1996; 14: 620-2.

4. Wagner JM, McKinNey WP, Carpenter JL. Does this patient have appendicitis? J Am Med Assoc 1996; 276: 1589-94.

5. Andersson RE, Hugander AP, Ghazi SH. Diagnostic value of disease history, clinical presentation, and inflammatory parameters in appendicitis. World J Surg 1999; 23: 133-40.

6. BeLtrán MS, ViLar RM, TAPia TF. Score diagnóstico de apendicitis: Estudio prospectivo, doble ciego, no aleatorio. Rev Chil Cir 2004; 56: 550-7.

7. Korner $H$, Sondenaa $K$, Soreide JA. The history is important in patients with suspected acute appendicitis. Dig Surg 2000; 17: 364-9.

8. Paulson EK, Kalady MF, Pappas TN. Suspected appendicitis. N Engl J Med 2003; 348: 236-42.

9. Rettenbacher T, Holerweger A, Gritzmann N. Appendicitis: Should diagnostic imaging be performed if the clinical presentation is highly suggestive of the disease? Gastroenterology 2002; 123: 992-8.

10. GronRoos JM, Gronroos P. Leukocyte count and C-reactive protein in the diagnosis of acute appendicitis. Br J Surg 1999; 86: 501-4.

11. RaO PM, RHEA JT, Novewne RA. Effect of computed tomography of the appendix on treatment of patients and use of hospital resources. N Engl J Med 1998; 338: 141-6. tentativo de apendicitis en consultorios rurales sin médico residente, consultorios generales, UE de clínicas y hospitales que no disponen de estudios radiológicos y en la práctica de cirujanos y médicos generales con poca experiencia clínica.

12. Schuler JG, Shortsleveve MJ, Goldenson RS. Is there a role for abdominal computed tomographic scans for appendicitis? Arch Surg 1998; 133: 373-7.

13. Barron B, Hanna C, Passalaqua AM. Rapid diagnostic imaging of acute, nonclassic appendicitis by leukoscintigraphy with sulesomab, a technetium 99m-labeled antigranulocyte antibody Fab' fragment. Surgery 1999; 125: 288-96.

14. OעaK D, Sinow R, French S. Computed tomography scanning for the diagnosis of perforated appendicitis. Am Surg 1999; 65: 959-64.

15. Weyant MJ, Eachempati SR, Maluccio MA. Interpretation of computed tomography does not correlate with laboratory or pathologic findings in surgically confirmed acute appendicitis. Surgery 2000; 128: 145-52.

16. Wilson EB, Cole JC, NipPeR ML. Computed tomography and ultrasonography in the diagnosis of appendicitis. Arch Surg 2001; 136: 670-5.

17. Mittal VK, Golath J, SabiR M. Advantages of focused helical computed tomographic scanning with rectal contrast only vs triple contrast in the diagnosis of clinically uncertain acute appendicitis. Arch Surg 2004; 139: 495-500.

18. Franke C, Bohner H, Yang Q, Ohmann C. Ultrasonography for diagnosis of acute appendicitis: Results of a prospective multicenter trial. World J Surg 1999; 23: 141-6.

19. Vaшina VL, Velasco JM, McCuLoch CS. Laparoscopic versus conventional appendectomy. Ann Surg 1993; 218: 685-92.

20. Slim K, Pezet D, Chippon J. Laparoscopic or open appendectomy? Critical review of randomized controlled trials. Dis Colon Rectum 1998; 41: 398403.

21. Moberg AC, Montgomery A. Introducing diagnostic laparoscopy for patients with suspected acute appendicitis. Surg Endosc 2000; 14: 942-7. 
22. GuLer U, Hervey S, Purves H. Laparoscopic versus open appendectomy outcomes comparison based on a large administrative database. Ann Surg 2004; 239: 43-52.

23. Pedersen AG, Petersen OB, Wara P. Randomized clinical trial of laparoscopic versus open appendicectomy. Br J Surg 2001; 88: 200-5.

24. KuRTZ RJ, Heimann TM. Comparison of open and laparoscopic treatment of acute appendicitis. Am J Surg 2001; 182: 211-14.

25. Piskun G, KoziK D, Rajpal S. Comparison of laparoscopic, open, and converted appendectomy for perforated appendicitis. Surg Endosc 2001; 15: 660-2.

26. Wuldtein C, Barkhausen S, Gross E. Results of laparoscopic vs conventional appendectomy in complicated appendicitis. Dis Colon Rectum 2001; 44: 1700-5.

27. FenYo G. Routine use of a scoring system for decision-making in suspected acute appendicitis in adults. Acta Chir Scand 1987; 153: 545-51.

28. Fenyo G, LindBerg G, Bund P. Diagnostic decision support in suspected acute appendicitis: Validation of a simplified scoring system. Eur J Surg 1997; 163: 831-8.

29. Ohmann C, Franke C, Yang Q. Diagnostic score for acute appendicitis. Chirurg 1995; 66: 135-41.

30. OHMAnN C, Yang Q, Franke C. Diagnostic scores for acute appendicitis. Abdominal pain study group. Eur J Surg 1995; 161: 273-81.

31. Ohmann C, Franke C, Yang Q. Clinical benefit of a diagnostic score for appendicitis: Results of a prospective interventional study. Arch Surg 1999; 134: 993-6.

32. Alvarado A. A practical score for the early diagnosis of acute appendicitis. Ann Emerg Med 1986; 15: 557-64.
33. MaLIK AA, Wani NA. Continuing diagnostic challenge of acute appendicitis: Evaluation through modified Alvarado score. Aust N Z J Surg 1998; 68: 504-5.

34. Zielke A, SitTer H, Rampp T. Clinical decisionmaking, ultrasonography, and scores for evaluation of suspected acute appendicitis. World J Surg 2001; 25: 578-84.

35. ESKELINEN M, IKONEN J, LIPPONEN P. A computerbased diagnostic score to aid in diagnosis of acute appendicitis: A prospective study of 1333 patients with acute abdominal pain. Theor Surg 1992; 7: 86-90.

36. Sitter H, Hoffmann S, Hassan I, Zielke A. Diagnostic score in acute appendicitis: Validation of a diagnostic score (Eskelinen score) in patients in whom acute appendicitis is suspected. Langenbecks Arch Surg 2004; 389: 213-18.

37. Bijnen CL, Van Der Broek WT, Bujnen AB. Implications of removing a normal appendix. Dig Surg 2003; 20: 215-21.

38. Douglas CD, McPherson NE, Douglas PM, Gani JS. Randomized controlled trial of ultrasonography in diagnosis of acute appendicitis, incorporating the Alvarado score. BMJ 2000; 321: 1-7.

39. Boyssut PM, Reitsma JB, Bruns DE. Towards complete and accurate reporting of studies of diagnostic accuracy: The STARD initiative. BMJ 2003; 326: 41-4.

Agradecimientos

A los enfermeros y enfermeras de la Unidad de Emergencias del Hospital de Ovalle, quienes han contribuido con su trabajo a la aplicación del score y a la recolección de la información para el presente estudio: Jazmín Rojas, Tomás Araya, Marcela Barraza, Elizabeth Olivares, Isabel Alfaro, Manuel Cáceres. 